\title{
College women's awareness and consumption of folic acid for the prevention of neural tube defects
}

John M. Quillin, MS, Judy Silberg, PhD, Pamela Board, MS, Lee Pratt, PhD, and Joann Bodurtha, MD, MPH

\begin{abstract}
Purpose: Awareness and consumption of folic acid in the context of neural tube defect prevention among college women were assessed. Methods: Subjects documented folic acid awareness and multivitamin consumption. Beliefs about folic acid before and after an educational intervention were characterized using the Health Belief Model (HBM) and the Fetal Health Locus of Control Scale (FHLCS). Results: Awareness of folic acid was not associated with multivitamin consumption. Belief variables were not associated with awareness or consumption of folic acid. Conclusion: This study does not support educational interventions based on the HBM or FHLCS to increase multivitamin consumption among college women. Genetics in Medicine, 2000:2(4):209-213.
\end{abstract}

Key Words: Folic acid, neural tube defects, health belief model, college, pregnancy

Neural tube defects result from incomplete closure of a fold of ectodermal cells on about the $28^{\text {th }}$ day of embryogenesis. This failed closure occurs in about 1.5 of every 1000 live-births and contributes significantly to neonatal morbidity and mortality. ${ }^{1}$ Within the last decade it has been found that regular, adequate periconceptional consumption of folic acid by women can significantly reduce incidence of neural tube defects (NTDs) in pregnancy. ${ }^{2-4}$ Currently in the United States, adequate daily folic acid consumption is considered to be 0.4 $\mathrm{mg}$ for all women capable of becoming pregnant. ${ }^{5}$ This recommendation is consistent with the Public Health Service (PHS) recommendation. ${ }^{6}$ Furthermore, in addition to preventing half of all NTDs, adequate folic acid consumption may also decrease the chance of preterm delivery and low birth weight. ${ }^{7}$

Previous studies indicate that many women are not aware of the preventive benefits of adequate folic acid consumption prior to pregnancy. A 1996 report, conducted by the Georgia Department of Human Resources, examined consumption of folic acid supplements among women in Georgia. ${ }^{8}$ This report demonstrated that only $29 \%$ of respondents claimed they had heard folic acid can help prevent birth defects. Of these women who had heard about folic acid, only $30 \%$ reported taking daily a multivitamin containing at least $0.4 \mathrm{mg}$ folic acid. Results from the most recent national survey, conducted July-August 1998 by the National March of Dimes Birth Defects Foundation and the Gallup Organization, suggest that $68 \%$ of women surveyed had heard or read about folic acid, and women ages 18-24 were least likely to have heard about folic acid. ${ }^{9}$ This survey also showed that only $32 \%$ of surveyed reproductive-age women daily consumed a supplement containing folic acid. Other studies show similar results. ${ }^{10,11}$ That knowledge of folic acid's benefits does not neces-

From the Virginia Commonuterlth (inirersity, Richmond, Virginia

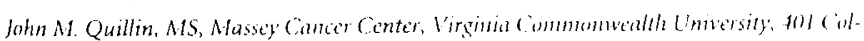
lege Stret. P.O. Box 980037, Richmond, V., 23298-1903.37.

Received: March 14, $2(0)$

Accepted: May 21, 2000. sarily lead to consumption is concerning. It would be valuable to identify variables from behavioral models that might account for this discrepancy between those women who know about folic acid's benefits, and those women who actually consume folic acid supplements daily.

The Health Belief Model traditionally identifies at least four variables as significant predictors of compliance with a given health regimen. ${ }^{12,13}$ These variables are a person's:

a) perceived susceptibility to suffering a health related consequence if $s /$ he does not comply with the suggested health regimen;

b) perceived seriousness of the health problem;

c) perceived benefit of the health regimen to overcome or to prevent the health problem; and

d) perceived barriers to complying with the health regimen.

Since its inception, other factors such as knowledge and locus of control have become part of the HBM. This model has been used to study a number of health practices including sunscreen application to prevent skin cancer, ${ }^{14}$ breast self-examinations, ${ }^{15}$ and diabetes self-care. ${ }^{16}$ Kloeblen ${ }^{17,18}$ characterized folate-related knowledge and behaviors of pregnant women of low socioeconomic status according to HBM variables. Folate consumption in Kloeblen's study was measured as a function of reported fortified grain intake and of periconceptional supplement use. Kloeblen found that preconceptionally using supplements and some indicators of knowledge were correlated with HBM variables, and she concluded that the HBM might serve as an effective framework for educational interventions among this group of women. 17.18

The Fetal Health Locus of Control Scale (FHLCS) has been validated as a means of using locus of control variables to characterize pregnancy-related health behaviors. ${ }^{19}$ The FHLCS includes 18 statements that characterize locus of control for maternal health behavior. Subjects completing the FHLCS evaluation are asked to indicate the extent to which they agree with each statement on a scale from one to nine. These 18 items cover three 
subcategories including internal controls (I), chance (C), and powerful others $(\mathrm{P})$. Alpha coefficients of reliability for each subscale are $0.88(\mathrm{I}), 0.83(\mathrm{C})$, and $0.76(\mathrm{P})$. Characterization of locus of control variables in relation to multivitamin consumption for NTD prevention could also be helpful in developing effective educational interventions aimed at increasing folic acid use among women of childbearing age.

For this study we decided to examine college women. College women are an important group to target for folic acid educational interventions for many reasons. The majority of the college population consists of young people (18-24 years old), and this age group is at highest risk for unintended pregnancy. 20.21 One study showed that on college campuses pregnancy rates were estimated to be between $6 \%$ and $10 \%{ }^{20} \mathrm{Re}$ gardless of unintended pregnancies, many of these future college graduates will at some point become parents. College campuses provide a unique opportunity to send messages about family planning and preconceptionally caring for a large number of young adults.

Vitamin supplementation might be the best means of incorporating folic acid in college women's diets. Synthetic folic acid has been shown to be a more effective means of increasing red-cell folate compared with consumption of naturally-occurring food folate."2- Furthermore, college students tend to eat fewer fruits and vegetables (good sources of folate) than recommended. ${ }^{23}$ Based upon the validity and reliability of this research, there is a need to promote vitamin supplementation among college women.

We, therefore, designed a study to characterize a sample of college women with regard to:

a) vitamin consumption practices;

b) knowledge of folic acid and of NTDs; and

c) associated behavioral variables.

Understanding college women's folic acid consumption practices and beliefs could lead to the design of more effective promotion campaigns. To test the hypothesis that HBM and FHLCS variables are not only associated with vitamin consumption practices but also can be changed, an intervention addressing the variables of the HBM was performed to assess changes in attitudes and knowledge.

\section{MATERIALS AND METHODS}

Following approval by Virginia Commonwealth University's (VCU) Committee on the Conduct of Human Research, women enrolled in the undergraduate psychology program at VCU were recruited to participate in the study through class announcements and flyers posted in the undergraduate psychology administrative building. This group of women was recruited as part of their undergraduate curriculum. All psychology students at $\mathrm{VCU}$ are required to participate in psychology research. The present study was one of several from which they could choose. Interested students signed up for one of four meeting times.
A total of 71 female students participar in the study. Signed informed consent was obtained from ...h of the study participants. Subjects met for 45-minute issions during which they completed pretests, listened to a five-minute educational slide presentation about folic acid, completed posttests, and were finally debriefed.

\section{Pretest}

The pretest questionnaire assessed subjects' awareness of folic acid and of neural tube defects, and documented their current multivitamin consumption practices. Beliefs about folic acid were characterized according to variables associated with the HBM and the FHLCS.

\section{Intervention}

Following completion of the pretests, subjects listened to a 5 minute educational slide-show. This presentation defined folic acid, NTDs, and the known association of increased folic acid consumption with decreased incidence of NTDs. Each variable of the HBM was addressed. The population incidence of NTDs was discussed along with the message that each subject is at risk if she is able to have children. Women were also told that, to be effective, folic acid must be taken regularly before a woman knows that she is pregnant. Furthermore, subjects were told that about $50 \%$ of pregnancies in the United States are not planned; thus, it is recommended that all women capable of pregnancy take folic acid daily. NTDs were characterized as serious birth defects. The benefits of folic acid consumption were stressed including prevention of NTDs, as well as decreased chance of heart disease ${ }^{2+}$ and some types of cancer. ${ }^{25}$ Convenience and inexpensive cost of folic acid supplements were emphasized. Additionally, the importance of synthetic folic acid supplementation was stressed in contrast to reliance on food folate as one's only source of folic acid.

\section{Post-test}

After listening to this presentation, each group of participants completed a post-test. The post-test was similar to the pretest except that it excluded questions that were not subject to change (demographic information, pregnancy history, etc.) Finally, each group was debriefed regarding the purpose of the study and the development of and theory behind the HBM. Each group was offered the opportunity to ask questions and was given a brochure that addresses the importance of folic acid supplementation at the end of the debriefing.

Data obtained from the questionnaires were analyzed using SAS version 6.12 (SAS Institute). From these analyses, we obtained a characterization of the subjects' current multivitamin supplementation use and their awareness of folic acid and of NTDs.

\section{RESULTS}

Subjects in the study ranged in age from 17 to 50 years. Sixty-seven $(94 \%)$ subjects were $<25$ years old. Twenty-three $(32 \%)$ subjects claimed they currently take multivitamin sup- 
plements, and 48 (68\%) subjects said they did not currently take supplements. Prior to the intervention, 49 (69\%) subjects reported having heard of NTDs, and $22(31 \%)$ subjects reported they had not heard of NTDs. Forty-six (64\%) subjects reported having heard about folic acid, although only $4(5 \%)$ subjects correctly identified $0.4 \mathrm{mg}$ as the recommended daily allowance (RDA) of folic acid.

Subjects were asked how likely, on a 5-point Likert scale, they were to become pregnant in the next year, and most felt that they were unlikely or very unlikely to become pregnant. Results from the pretest analysis (Fig. 1) show that $94 \%$ of subjects felt they were either unlikely or very unlikely to become pregnant in the next year, and these perceptions did not change significantly from pretest to post-test. No significant association was found between perception of pregnancy likelihood and having heard of folic acid $\left(\mathrm{X}^{2}=2.078, P=0.556\right)$ nor was there an association between perception of pregnancy likelihood and multivitamin consumption $\left(\mathrm{X}^{2}=1.624, P=0.654\right)$.

Prior to the intervention, there was a significant association among women who had heard of folic acid and those who had heard of neural tube defects $\left(\mathrm{X}^{2}=5.223, P=0.022\right)$. However, on pretest there was no significant correlation between those who currently take vitamin supplements, and those individuals who had heard of folic acid $\left(\mathrm{X}^{2}=2.707, P=0.100\right)$ (Fig. 2).

\section{Health belief model variables}

Factor analysis was performed on pretest survey responses to determine which questions would be used for each of the expected health belief model variables. Although four catego-

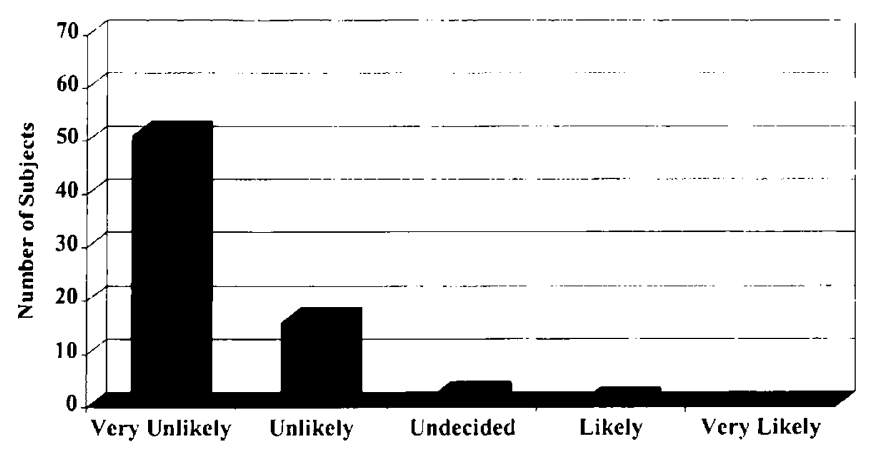

Fig. 1 Perceived likelihood of pregnancy in the next year.

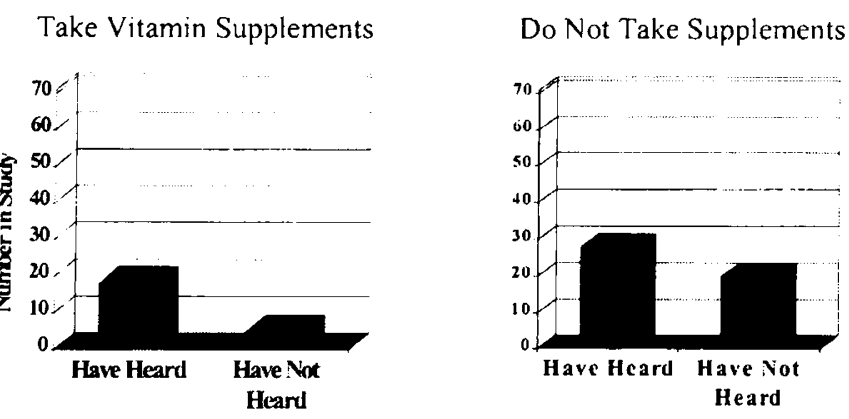

Fig. 2 The association between having heard of folic acid and reported consumption of multivitamins. $\chi^{2}=2.707, P=0.100$. ries of questions were anticipated, factor analysis revealed three categories of questions that accounted for the most variance. One category, heretofore referred to as "perceived threat," incorporates both questions about perceived seriousness of NTDs and subjects' perceived susceptibility to having a child with an NTD. Questions shown to explain variance in responses are as follows:

\section{Perceived benefits}

B1. Even if she is not planning a pregnancy every woman capable of becoming pregnant should take folic acid supplements.

B2. Taking folic acid prior to pregnancy significantly lowers my chances of having a child with a neural tube defect.

B3. It is important for women considering pregnancy to consume folic acid each day in order to prevent neural tube defects.

$\mathrm{B} 4$. Is folic acid necessary to prevent neural tube defects in pregnancy?

B5. It is easy to consume the recommended amount of folic acid through vitamin supplements.

\section{Perceived barriers}

R1. I don't need to take folic acid supplements because I get enough in my diet.

R2. Vitamin supplements that contain folic acid are easy to find. R3. Vitamin supplements are affordable.

$\mathrm{R} 4$. If someone does not have a family history of neural tube defects, she is not at risk for having a baby with a neural tube defect.

\section{Perceived threat}

S1. If I had a child born with a neural tube defect I would consider placing the child out of the home to live (examples: foster care, adoption, etc.)

S2. If you are not planning a pregnancy, how likely are you to become pregnant in the next year?

S3. If I had a child born with a neural tube defect this would be a serious concern to me.

These modified HBM variables, along with those associated with the FHLCS, were then used to analyze behavior and perceptions.

\section{Pretest analyses}

Relationships between belief variables and awareness of folic acid and NTDs as well as multivitamin consumption are displayed in Table 1. Subjects who had heard about folic acid were more likely to believe in the benefits of folic acid compared with those who had not heard of folic acid $(P<0.01)$. Likewise, subjects who had heard about folic acid perceived barriers to taking folic acid to be easier to overcome $(P<0.05)$. No other significant differences were identified with respect to any other belief variables (HBM or FHLCS) among those who were and were not aware of folic acid. With regard to any belief variables, no significant differences were found between those who did 
Table 1

Student t-test analysis of the relationship between belief variables and three knowledge/behavioral variableshearing about folic acid, hearing about neural tube defects, and taking multivitamin supplements ${ }^{a}$

\begin{tabular}{|c|c|c|c|}
\hline & Heard of Folic Acid & $\begin{array}{c}\text { Heard of Neural Tube } \\
\text { Defects }\end{array}$ & $\begin{array}{l}\text { Take Multivitamin } \\
\text { Supplements }\end{array}$ \\
\hline & $\begin{array}{c}\mathrm{t} \text {-test }(\mathrm{df}=24) \\
P \text { value }\end{array}$ & $\begin{array}{c}\text { t-test }(\mathrm{df}=21) \\
P \text { value }\end{array}$ & $\begin{array}{c}\text { t-test }(\mathrm{df}=22) \\
P \text { value }\end{array}$ \\
\hline \multicolumn{4}{|l|}{ Health Belief Model } \\
\hline Perceived Benefits & $2.48,<0.01$ & $1.38, \mathrm{~ns}$ & $1.02, \mathrm{~ns}$ \\
\hline Perceived Barriers & $2.32,<0.05$ & $1.15, \mathrm{~ns}$ & $1.23, \mathrm{~ns}$ \\
\hline Perceived Threat & $0.43, \mathrm{~ns}$ & $0.17, \mathrm{~ns}$ & $1.11, \mathrm{~ns}$ \\
\hline \multicolumn{4}{|c|}{ Fetal Health Locus of Control Scale } \\
\hline Powerful Others & $1.59, \mathrm{~ns}$ & $0.05, \mathrm{~ns}$ & $0.86, \mathrm{~ns}$ \\
\hline Chance & $0.06, \mathrm{~ns}$ & $0.20, \mathrm{~ns}$ & $0.98, \mathrm{~ns}$ \\
\hline Internal Control & $0.78, \mathrm{~ns}$ & $0.57, \mathrm{~ns}$ & $0.67, \mathrm{~ns}$ \\
\hline
\end{tabular}

"Within the table " $\mathrm{df}$ " indicates degrees of freedom, and "ns" indicates a non-significant $P$ value $(P>0.05)$

and did not consume multivitamins regularly, nor among those who had or had not heard about NTDs.

\section{Post-test analyses}

Changes in post-test responses compared with pretest responses were determined using the student's t-test. Following the intervention, a significant increase in knowledge of both folic acid $(P=0.0001)$ and of NTDs was found $(P=0.0002)$, and there was a significant increase in scores for the perceived benefits factor $(P=0.0001)$, for the perceived barriers factor $(P=0.0001)$, and for the perceived threat factor $(P=0.0001)$. There was not a significant change in scores for any of the subscales of the FHLCS (internal control, $P=0.615$; chance, $P=0.470$; powerful others, $P=0.151$ ).

\section{DISCUSSION}

The current study may serve as a promising exploration into behavioral variables that influence a woman's decision to consume folic acid through multivitamin supplements even when she is not contemplating pregnancy. Pretest analyses demonstrated a significant association between awareness of folic acid and awareness of NTDs. Women who knew about one were more likely to know about the other. It is not clear, however, whether these women know that folic acid prevents neural tube defects. It might be the case, for example, that women who know about folic acid are more knowledgeable about medicine in general and, thus, are more likely to know about NTDs.

Importantly, belief variables related to folic acid were not associated with multivitamin consumption. Thus, subjects who currently take vitamin supplements do not appear to do so because of beliefs explored in this study nor do they consume vitamin supplements because of an awareness of folic acid or of NTDs. These findings might have important implications for constructing effective educational interventions. Many current campaigns to increase folic acid consumption focus on education about the preventive effects of daily maternal folic acid use. Our data do not predict the success of such campaigns in a college population.

In particular, locus of control variables were not associated with multivitamin consumption (powerful others, $P=0.439$; chance, $P=0.352$; internal control, $P=0.481$ ). In other words, subjects' motivation to take multivitamins containing folic acid was not related to their beliefs in their abilities to influence the outcome of pregnancy. This finding is further supported by the lack of an association between fetal health locus of control and having heard about folic acid (powerful others, $P=0.154$; chance, $P=0.950$; internal control, $P=$ 0.400 ). Both of these findings and the general disbelief in pregnancy susceptibility (see Fig. 1) suggest that these subjects are not focused on preconceptional care.

This suggestion is not trivial considering the relatively high rate of pregnancy reported on college campuses, and the high rate of unintended pregnancy in general. Ninety-four percent of the women in our study felt they were unlikely or very unlikely to become pregnant in the next year. However, this study did not explore variables specifically associated with this belief. Other characteristics of the study population such as sexual activity, method and frequency of birth control, sexuality, etc. will be important to consider in future studies before conclusions about this belief can be drawn.

However, we did not find any association between perception of pregnancy susceptibility and multivitamin consumption, although larger numbers of subjects might better clarify this relationship. It might be that an increase in multivitamin consumption (and thus folic acid consumption) among college women will occur more easily through education about other health benefits related to general vitamin intake. In other words, women who take multivitamin supplements might simply believe in the efficacy of vitamins generally. In any case, our results point to the need for greater exploration of motivators before investing in folic acid campaigns targeted toward this group. 
Because this study was exploratory, it was limited by relatively small numbers, so a need for larger studies in more diverse populations is recognized. Despite these limitations, our findings encourage educators to think broadly about effective means of increasing folic acid consumption preconceptionally. Our study found that, although educational interventions might prove effective at increasing belief in the efficacy of folic acid toward NTD prevention, our characterization of this population suggests that such an increase will not necessarily lead to more folic acid consumption through multivitamins.

It is important to note, however, that analysis of our study involved a modification of the HBM. Although in general each of the variables designed to reflect a particular health-related belief were categorized appropriately, two specific questions did not follow this pattern. Question B5, "It is easy to consume the recommended amount of folic acid through vitamin supplements," was designed to capture perceived barriers. However, factor analysis suggests responses were consistent with variables designed to capture perceived benefits. Likewise, question R4, "If someone does not have a family history of neural tube defects, she is not at risk for having a baby with a neural tube defect," was demonstrated by factor analysis to be consistent with variables associated with a perceived barriers category rather than a perceived susceptibility category. Both findings highlight the fact that the HBM, although validated as predictive for many health behaviors, is a theoretical construct rather than a rule to which behaviors and/or beliefs must adhere. In any case, the findings indicate the need for reanalysis of a similar population using different variables for clarification.

For purposes of analysis, two aspects of the HBM-perceived seriousness and perceived susceptibility-were combined to serve as one belief variable deemed "perceived threat." This combination was chosen based on factor analysis that suggested that these two belief variables overlapped in the studied population. Although not traditionally a part of the HBM, this merged category of perceived threat has precedence in other research using the HBM. ${ }^{13}$ Still, given that we did not find predictive relationships between belief variables and multivitamin consumption, a more thorough examination of HBM variables with respect to vitamin consumption in college women is indicated. Different survey questions specific to seriousness and susceptibility, for example, may demonstrate a distinction in subjects' responses. Future research with this population might benefit from a more expanded exploration of this phenomenon.

Due to logistical constraints we did not verify actual vitamin consumption following the intervention. However, if vitamin consumption did increase following the intervention, and if our modified HBM is predictive, data do not indicate that improvements in our measured belief variables would be responsible for such an increase. In other words, because belief variables are not shown to be associated with multivitamin consumption to begin with, we have no evidence to suggest that altering subjects' HBM-related beliefs would affect multivitamin practices.
It would be of value to conduct a study similar to the one presented using various modes of interventions and a control group that does not receive an educational intervention until after the post-test is administered. Furthermore, subjects' lack of concern for preconceptional issues even among those consuming folic acid regularly is notable. Thus, it might be useful to explore further college women's motivation for consuming vitamins in general. Subsequent design of educational interventions targeting these motivations might prove to increase effectively, though incidentally, folic acid use among these women.

\section{References}

1. Baty B, Cohen L, Phelps L, Speer M, Stengel P, Williamson-Kruse L. Folic acid and the prevention of neural tube defects: a position paper of the National Society of Genetic Counselors. J Genet Counseling 1996;5:139-145.

2. Berry RJ, Li Z, Erickson ID, Li S, Moore CA, Wang H, Mulinare I, Zhao P, Wong LY, Gindler J, Hong SX, Correa A. Prevention of neural-tube defects with folic acid in China: China-U.S. Collaborative Proiect for Neural Tube Defect Prevention. N Engl JMed 1999:341:1485-1490.

3. MRC Vitamin Study Research Group. Prevention of neural tube defects: results of the medical research council vitamin study. Innct 1991;338:131-137.

4. Czeizel AE, Dudas I. Prevention of the first occurrence of neural-tube defects by periconceptional vitamin supplementation. N Engl/ Med 1992;327:1832-1835.

5. Stevenson RE, et al. Prevention program for reducing risk for neural tube defectsSouth Carolina, 1992-1994. MNWR Morb Nortal Wkly Rep 1995;44:141-142.

6. Center for Disease Control. Recommendations for the use of folic acid to reduce the number of cases of spina bifida and other neural tube defects. MMWR M forb Mortal IVkly Rep 1992;41(RR-14):1-7.

7. Scholl T, Hediger M, Schall J, Khoo C, Fischer R. Dietary and serum folate: their influence on the outcome of pregnancy. Am J Clin Nutr 1996;63:520-525.

8. Serbanescu F, Rochat R, Floyd V, Toomey KE. Knowledge about folic acid and use of multivitamins containing folic acid among reproductive-aged women-Georgia, 1995. MMIVR Morb Mortal Wkly Rep 1996:45:793-795.

9. Petrini IR, Damus K. Johnston RB, Mattison DR. Knowledge and use of folic acid by women of childbearing age - United States, 1995 and 1998. MMIVR Morb Mortul Whly Rep 1999;48:325-327.

10. Oakley GP. More folic acid for everyone, now. J Nutr 1996;126:751S-755S

11. Broome $K$. Folic acid campaign and evaluation-Southwestern Virginia, 1997-1999. MMIVR Morb Mortal Wkly' Rep 1999:48:914-917.

12. Rosenstock IM, Strecher VJ, Becker MH. Social learning theory and the Health Belief Model. Health Edtui Q 1988;15:175-183.

13. Strecher VI, Rosenstock IM. The health belief model. In: Glanz K, Lewis FM, Rimer $B K$, editors. Health behavior and health education: theory, rescarch, and practice, 2nd Ed. San Francisco: Iossey-Bass Publishers; 1997:41-59.

14. Marlenga $B$. The health beliefs and skin cancer prevention practices of Wisconsin dairy farmers. Oncol Nurs Forum 1995;22:681-686.

15. Champion VL. Use of the Health Belief Model in determining frequency of breast self-examination. Res Nurs Health 1985:8:373-379.

16. Hurley CC, Shea CA. Self-efficacy: strategy for enhancing diabetes self-care. Diabletcs Educ 1992:18:146-150.

17. Kloeblen AS. Folate knowledge, intake from fortified grain products, and periconceptional supplementation patterns of a sample of low-income pregnant women according to the Health Belief Model. I Am Diet Assoc 1999:1:33-38.

18. Kloeblen AS, Batish SS. Understanding the intention to permanently follow a high folate diet among a sample of low-income pregnant women according to the Health Belief Model. Health Educ Res 1999;14:327-338.

19. Labs SM, Wurtele SK. Fetal Health L.ocus of Control Scale: development and validation. J Consult Clin Psychol 1986;54:814-819.

20. Sawyer RG, Pinciaro PJ, Anderson-Sawyer A. Pregnancy testing and counseling: a university health center's 5-year experience. I Am Coll Hialth 1998:46:221-225.

21. Henshaw SK. Unintended pregnancy in the I'nited States. Fam Plann Perspect 1998; $30: 24-31$

22. Cuskelly GI, MCNulty H, Scott IM. Effect of increasing dietary folate on red-cel folatc: implications for prevention of neural tube defects. Lancet 1996:347:657-659.

23. Johnston CS, Solomon RE. Corte C. Vitamin C status of a campus population: college students get a C minus. / Am Coll Health 1998;46:209-213.

24. Boushey CI, Beresford SAA, Omenn CS, Motulsky AG. A quantitative assessment of plasma homocystcine as a risk factor for vascular disease. JMMA 1995;274:1049-1057.

25. Mason IB, Levesque T. Folate: effects on carcinogenesis and the potential for cancer chemoprevention. Oncol 1996;10:1727-1742 